\title{
sis \\ Personalized Image Search using Hybrid Re-ranking Based on Ontology
}

\author{
Chaitali Jambhulkar, Ankita Borana, Chaitali Deshmukh, Priyanka Nanaware \& Chandrama G. Thorat \\ Department of Computer, Rajarshi Shahu College of Engineering, Pune-411033, India
}

\begin{abstract}
Now a day's use of internet is increased with a big extent due to this large data is uploaded and downloaded by various internet users and a quite large-scale of data is processed. Users can create, share and comment Medias using social sharing websites like Flickr and YouTube so once we have such a huge data to handle on the internet we cannot find the right information which we are searching. The data generated by user called metadata provides multiple things like sharing, organizing multimedia contents and provides useful information related to multimedia management. Due to this reason we are proposing a "Personalized image search Engine by using ontological search and hybrid reranking Algorithms". By using this algorithm we can make our search personalized with a specific limit. This system is advantageous over Google because Google gives nonpersonalized information, which is not as per user desirable requirements. The basic premise of our system is to combine user preferences and query related search into user specific topics. This proposed system consists of two parts, first part contains hybrid re-ranking algorithm based on ontology about images as well as text and second part contains ontological user profiles for personalized image search.
\end{abstract}

Keywords - Multimedia, Ontology, Personalization, Re-ranking.

\section{INTRODUCTION}

This section gives the information regarding software and hardware requirements for the system which we have built. This section also gives the operating environment needed for working of this software.

Development of web and its influence on the people and next generation is increasing rapidly. The most common activity done on the internet involves the image search. Searching for images related to a person accounts for more than five percent of the current image searches. Image search is difficult because it is hard for users to construct queries that are both sufficiently descriptive and sufficiently discriminating to find just the web images that are relevant to the user's search goal.

Queries are often ambiguous; this will lead to search result containing different images. Often the user is unable to understand the obtained result. For this the user must refine the query in order to get the required image, but this is a time consuming process. Personalize image search is an approach which refines the query and presents the user with required result. However, the result returned are in the form of ranked list images along with partial content leaving the job of finding the specific document onto the user.

Our approach exploits the task of searching the image in more descriptive manner. The personalize search engine reranks the images related to the query fired by the user. A hybrid re-ranking approach [2] for personalized search system that reranks search results based on user ontological profiles and combines the information in all documents to provide personalized search results. Each concept in ontological user profile is build which is associated with three different documents: ontology, positive and negative documents.

Figure 1 shows the two main phases. First phase is the building ontological user profile [3] and second one is the personalized search process. Ontological user profile includes collecting user browsing behavior, preparing the reference ontology, learning and constructing the user profile and finally building the ontological user profile. The second phase is the search personalization process which involves retrieving search results based on user queries and then processing these results with regard to users' ontological profiles in order to provide personalized search results. In this phase the hybrid re-ranking algorithm is applied on collected data that is positive documents, negative documents and documents from reference ontology. Then correct data is retrieved based on original search results. 


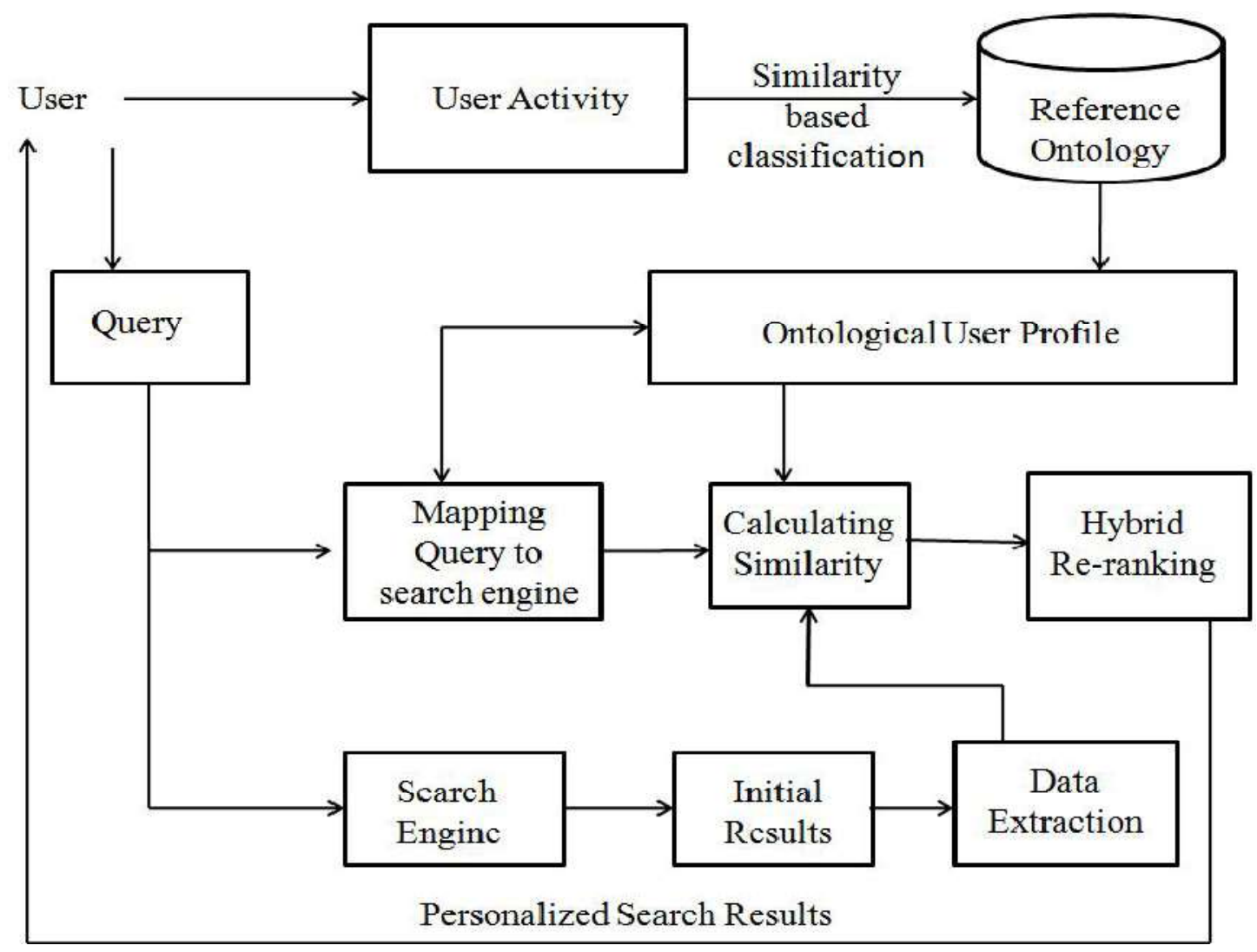

Fig. 1 : Proposed System

\section{OVERVIEW OF THE APPROACH}

In this section, we give an overview of all important algorithms and components, which are necessary to implement the system. We will use the middleware approach to our system. In our proposed approach the processing of query consists of steps as follows:

\section{Input:}

The user submits the query related to image he want to search to the middleware through the web-based interface.

\section{Retrieval of images:}

The middleware [5] then checks whether the query provided by the user is valid or invalid by the process of filtering. If query is valid i.e. the query is for searching a image, then it queries the search engine with the same query provided by user. It then retrieves the top relevant images from the search engine.

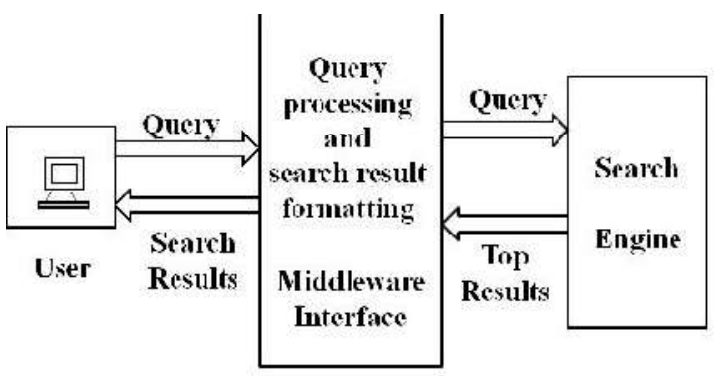

Figure2. Retrieval of images

\section{Reference Ontology:}

Many studies have utilized ontology to shown more effective personalization systems. In this paper, ontology plays a key role in modeling the user profile. Reference ontology provides a clear illustration of contents of a particular domain of application. Unlike flat representations, a reference ontology provides a richer representation of information in that semantic and structural relationships are defined explicitly. In this paper, the user profile is modeled and user interests are generated from the Open Directory Project (ODP) reference ontology. ODP is one of the largest manually constructed directories which consist of a hierarchical ontology of websites categorized in related topics.

Our current implementation uses the Open Directory Project, which is organized into a hierarchy of topics and Web pages that belong to these topics. We utilize the Web pages as training data for the representation of the concepts in the reference ontology. The textual information that can get extracted from Web pages explains the semantics of the concepts and is learned as we build a term vector representation for the concepts.

\section{Ontological user profile:}

For constructing ontological user profile following parts needs to be considered: 
1. User behavior: In user behavior some information about the user and interest of the user is collected. User information collected by implicitly and explicitly. Most of the time user information is collecting implicitly. Information is nothing but spending time on that page and visited pages. And store this information in file.

\section{Constructing ontological profile:}

Ontological user profile is created by three documents. Ontology documents which contains information which is same as reference ontology. Next document is positive document contains information which is obtained by interesting visited pages of user. And third document is negative document contains information which obtain by uninteresting visited pages of user.

\section{ALGORITHM IMPLEMENTATION}

\section{A) User Interest Model:}

\section{1) Description of a User Interest Model:}

User interest model is the formalized description of the user's interest information.

It is calculated as follows:

Interest $_{\mathrm{i}}=\left\{\left(\mathrm{k}_{1}, \mathrm{w}_{1}\right), \ldots \ldots,\left(\mathrm{k}_{\mathrm{n}}, \mathrm{w}_{\mathrm{n}}\right)\right\}$

where, Interesti represents the interest model of user $U_{i}$., $\mathrm{K}_{\mathrm{i}}$ is the $\mathrm{i}$-th keyword which can be both extracted from the user's logs, queries and typed in by the user in advance and $\mathrm{W}_{\mathrm{j}}$ is the weight of keyword $\mathrm{K}_{\mathrm{i}}$.

2) Updation of User Interest Model:

It will update the user interest model dynamically. When a user $U_{j}$ send a query $k_{j}$, it will first find out whether the keyword $k_{j}$ is in his/her interest model. If the item $\left(\mathrm{k}_{\mathrm{j}}, \mathrm{W}_{\mathrm{j}}\right)$ is in Interesti, a unit score is added to $\mathrm{Wj}$. Otherwise a new item $\left(\mathrm{k}_{\mathrm{j}}, \mathrm{w}_{\mathrm{j}}\right)$ will be added into Interesti where $\mathrm{Wj}$ is the default value.

3) Compute the User interest Value:

It will be calculated as follows:

Input : uk' s interest model Interestk, result rj Output :Interest value Ikj of Uk to rj

Process:

$\mathrm{I}_{\mathrm{kj}}=0$

For each $\left(\mathrm{k}_{\mathrm{i}}, \mathrm{w}_{\mathrm{i}}\right) \in$ Interest $_{\mathrm{k}}$

if $\mathrm{r}_{\mathrm{j}}$ contains $\mathrm{k}_{\mathrm{i}}$

$\mathrm{I}_{\mathrm{kj}}=\mathrm{I}_{\mathrm{kj}}+\mathrm{w}_{\mathrm{i}}$

Return $\mathrm{I}_{\mathrm{kj}}$

\section{B) Collaborative Filtering Model:}

Assume $\mathrm{U}$ is the set of all the users. For each $u_{i} \in U, R_{i}$ is the set of the resources (search results) that $u_{i}$ had clicked,

$$
R=\bigcup_{U \in U} R_{i}
$$

So we obtained the matrix as shown in following figure:

\begin{tabular}{|l|l|l|l|}
\hline items & $\mathrm{r}_{1}$ & $\ldots$ & $\mathrm{r}_{\mathrm{n}}$ \\
\hline $\mathrm{u}_{1}$ & $\mathrm{M}_{11}$ & $\ldots$ & $\mathrm{M}_{\mathrm{in}}$ \\
\hline$\ldots$ & $\ldots$ & $\mathrm{M}_{\mathrm{ij}}$ & $\ldots$ \\
\hline $\mathrm{u}_{\mathrm{m}}$ & $\mathrm{M}_{\mathrm{m} 1}$ & $\ldots$ & $\mathrm{M}_{\mathrm{mn}}$ \\
\hline
\end{tabular}

Figure3. Recommendation matrix of users to items.

In the matrix, $u_{i} \in U$ and $r_{i} \in R$ Where $M_{i j}$ is the recommendation value of user $U_{i}$ to item $r_{j}$. In this paper we take the count of clicks as the recommendation value[4]. If user $U_{i}$ has never clicked item $r_{j}, M_{i j}$ will be set as

0. And then similarities of two users is calculated using cosine similarity.

For each user $u_{i}$, we compute set of its nearest neighbors as $C_{i}$ and its recommendation value $R_{i j}$ to the item $r_{j}$ based on $C_{i}$. Then we record the value $R_{i j}$ the $i$-th row and $j$-th column of matrix $M_{\text {out }}$.

$$
R_{i j}=\left\{\begin{array}{l}
M_{i j} \text { if } M_{i j} \neq 0 \\
P_{i j} \text { if } M_{i j}=0
\end{array}\right.
$$

When user u sends a search query, we first get the search results and for each result we check the corresponding recommendation value in the matrix $M_{\text {out. If }}$ $r_{j}$ is not in matrix $M_{\text {out }}$ then its recommendation value will be set as 0 .

\section{C) Personalization and re-ranking:}

Here we combined two models user interest and collaborating filtering model. Here the result is known as score and calculated as follows:

$$
\text { Score }_{i j}=\alpha * I_{i j}+(1-\alpha) * R_{i j}
$$

Where, $\alpha$ is the value of user logs in period T.

Then finally we arrange the score in decreasing order of Score $i j$ and obtain the final results. 


\section{CONCLUSION AND FUTURE} SCOPE:

In our web based days, the internet is the most important source of information. Search engines, try to provide the solution to user's problem, by providing them opportunity to specify a query and get the images that satisfy more of their parameters.

However, it is most complicated for the user to select the image that returned them among the results. We have introduced a personalized image search system that helps user to find images based on individual preferences. Our system also shows that, while it is possible to improve the efficiency of search using ontology method discussed above. In the future, the performance of engine may be improved by conducting large scale experiments.

\section{REFERENCES}

[1] JitaoSang, ChangshengXu, Senior Member, IEEE, DogyuanLu, "Learn to Personalized Image search From the Photo Sharing Website" IEEE transactions on multimedia, vol.14,no.4,August2012, pp. 963-973.

[2] Ahmad Hawalah and Maria Fasli "A Hybrid Reranking Algorithm Based on Ontological User Profiles", 3rd Computer Science and Electronic Engineering Conference, IEEE, 2011, pp.50-55.
[3] AhuSieg, Bamshad Mobasher and Robin Burke, "Ontological User Profiles for PersonalizedWeb Search", American Association for Artificial Intellingence, 2007.

[4] XiyuanWu,YanFu,ShixinTian,FengTinn, “A Hybrid Approach to personalized WebSearch",16th International Conference on Computer Supported Cooperative work inDesign,IEEE,2012, pp.214-220.

[5] S. Sendhilkumar,T.V.Geetha, "Personalized Ontology for Web Search Personalization", $A C M$,Banglore, Karnataka, India, 2008.

[6] S. Xu, S. Bao, B. Fei, Z. Su, and Y. $\mathrm{Yu}$,"Exploring folksonomy for personalized search,"inProc. SIGIR, 2008, pp. 155-162.

[7] Yun Zhu, Li Xiong, Christopher Verdery, "Anonymizing User Profiles for Personalized WebSearch", $A C M$, Raleigh, North Carolina, USA, 2010.

[8] Jayanthi J., Dr. K.S.Jayakumar, SruthiSurendran, "Generation of Ontology based User Profiles for Personalized Web Search", IEEE, 2011, pp.240244. 